\title{
Application of Dielectric Soil Moisture Sensors for Real-Time Automated Irrigation Control
}

M.C. Kuyper and J. Balendonck

Institute of Environmental and Agricultural Engineering (IMAG-DLO), department of Instrumentation and Measurement Technology, PO Box 43, 6700 AA Wageningen

The Netherlands

Keywords : leaching, flushing, temperature dependence, calibration methods

\begin{abstract}
In this article the irrigation control system to be developed in the running Waterman project is outlined. Also, the following process monitoring considerations are discussed: what soil moisture state to measure, calibration for soil water content, and the temperature dependence of soil dielectric constant and bulk electrical conductivity (EC).
\end{abstract}

\section{INTRODUCTION}

This preview article deals with the application of soil moisture sensors in the Waterman project. Because the difficulties encountered are a direct result of the specific application, the application is outlined first.

Leaching of nutrients and shortage of high quality irrigation water are two major problems in plant production systems. In arid areas the amount of high quality irrigation water is limited. Therefore growers want to use the available water as efficiently as possible. In the semi-arid regions of Northern Europe greenhouse plant production can be characterised by high productivity in combination with the use of large amounts of water and nutrients. It is common to grow with $50 \%$ surplus drain and to flush when too much salt accumulates. A large part of all drainage water containing the unused nutrients ends up in the surface water.

In 1989 the Dutch government has accepted a law to reduce emission of nutrients in order to protect the environment. By the year 2000 almost all greenhouse plant production will take place in closed systems, of which $30 \%$ will recirculate drainage water (Anonymous, 1989). For a number of crops, however, making the investments for new growing systems can not be done cost effectively. It is for the soil-bound, nonrecirculating plant production systems that Waterman wants to develop an automated irrigation control method that minimises water use and minimises leaching.

The key approach is keeping the water available in the root zone. A dry layer will be kept between the wet root zone and the water table, making the system virtually closed. The method to achieve this situation is to apply small amounts of water frequently. In this way the soil is given enough time to absorb the water instead of draining it immediately. Minimised leaching creates a new problem: salinization. To overcome the negative consequences of too saline soil the grower has to flush the accumulated salts every now and then. Waterman aims at developing a method for controlled flushing.

\section{CONTROL SYSTEM DESIGN}

\section{Basic Set-Up and Design Considerations}

Ideally an irrigation control system should: 1) use water efficiently, 2) minimise leaching, 3) be real-time, 4) be automated, 5) have a facility to flush when to many salts have accumulated, 6) be able to indicate the amount of leached water and nutrients, 7) need as little input data as possible, 8) be able to maintain a constant soil moisture level, 9) be cost effective and 10) be easy to operate. The idea behind the $8^{\text {th }}$ demand is that the precise soil moisture level for optimal crop growth is not that important, but rather that it stays at a constant level at a constant place (Boonekamp, 1994). In that way the root 
system does not have to put a lot of energy in constantly seeking water.

From a control theory point of view one can characterise the system on the basis of a basic closed-loop feedback control system. There is the process to control, a device that measures the process state, a controller that compares the process state with a setpoint and then calculates what the control action should be and an actuator that controls the process. For an irrigation control system the aforementioned system components are respectively: the soil water status, soil moisture sensors, the irrigation controller and the watering system (see Fig. 1). Water distracting and delivering mechanisms like evapotranspiration, upward and downward seepage can be considered as disturbances of the process, so these difficult mechanisms need not be modelled. Therefore the described irrigation control system relies heavily on input by soil moisture sensors. For an advanced control system to work properly it is vital to know how the process works and how it can be monitored. Difficulties encountered in this field are discussed in section 3.

\section{Available Technologies}

A survey of the available literature revealed one article describing a similar control theory method for surface irrigation (Reddy, 1985). Most irrigation methods described are solely based on e.g. crop water use models (Valiente et al., 1996), soil moisture sensor signals (Meron, 1995 and Anonymous, 1997) or infiltration estimation (Smith, 1996). A combination of some of the aforementioned techniques is applied by Thomson et al (1996) and by Hess (1996). None of these techniques meet all ten criteria mentioned in section 2.1.

The best technique available seems to be using tensiometers in combination with threshold values for starting an irrigation event. The principle is simple and flexible. With a tensiometer threshold controlled system the grower can reduce water use and leaching. Also, the system can run real-time and automated, needs no further input data, is able to maintain a constant soil moisture level, needs little investments, and is easy to operate. However, no optimisation routines are involved to really minimise water use and minimise leaching. Furthermore no tools are provided to control flushing and to indicate the amount of salts leached.

\section{The Final Set-Up}

The Waterman system will make use of the tensiometer threshold technique in an ordinary feedback control loop. This control loop forms the core, the inner loop, of the irrigation system. The two-optimisation routines, one for water use and one for leaching, will be connected to the core control loop as an outer control loop each (Fig. 2). The same applies for the flush control loop. The only variable available to be manipulated by the irrigation controller is the water supply: when and how much should we irrigate? No controlling of the amount of nutrients in the irrigation water will take place. The controlled variables for the process of soil moisture can be summarised as follows:

- $\quad$ irrigation timing and amount (input)

- $\quad$ level of leaching (output)

- $\quad$ level of water use (output)

- $\quad$ soil moisture level (output)

- $\quad$ level of salinization (output)

A two-dimensional ground water flow model (2D-HYDRA) will be available online to be used by the optimisation routines when necessary. For example, the leaching optimisation routine will use data of recent soil moisture measurements to compute the leaching that took place during that measurement period. If necessary it can then adjust the soil moisture set points used by the inner control loop. Because the dynamic properties of ground water flow are highly non-linear, it is important that these model properties are known accurately. To account for this necessity a control loop that finetunes these model properties may be added. This can be done in a similar way, the leaching algorithm uses recently measured data. The inner loop, which will be executed continuously, will have a schedule for calling the three-optimisation routines (minimum 
leaching, minimum water use, and model parameters adjustment). The EC level will be monitored by the inner loop. As soon as an upper limit is reached the flush control algorithm is given permission to take over the irrigation control. After the flush control algorithm has realised a pre-set EC value the inner loop will regain control over the irrigation.

\section{PROCESS MONITORING CONSIDERATIONS}

\section{Soil Moisture State to Measure}

In ground water flow there are two state variables: pressure head (h) and water content $(\theta$ for volumetric or $\mathrm{w}$ for gravimetric water content). When considering what variable to measure it is important to realise that with presently available sensors there are large differences in performance. Tensiometers have distinct other performance capabilities than water content sensors. Measuring pressure head is most common, because it can be measured directly and because conventional tensiometers are cheap and easy to operate. Measuring water content real-time can only be done through an indirect method, which is measuring another soil property that correlates strongly with soil water content. One of the possible properties eligible for measurement is the soil dielectric constant, which can be measured by means of a frequency domain (FD) or a time domain reflectometry (TDR) technique. To obtain a soil water content value from dielectric soil measurements one has to perform a soil specific calibration in advance. In applications where soil characteristics, like density changes with time or spatial variability, are large it is not possible to obtain high accuracy measurements based on one calibration. Although calibration of soil water content sensors can be considered a disadvantage, they have a larger working range and respond instantly to soil moisture changes. For plain irrigation control, the most probable cost-effective approach is based upon using tensiometer signals (see section 2.2). However, in the medium wet area of the water retention curve tensiometers do not always have as much resolution as water content measurements do (see Fig. 3),

Further, available tensiometers have a large response time to changes in pressure head while water content sensors give the exact value instantly. Thus, for accurately calibrating and/or validating a ground water flow model one might consider measuring both pressure head and water content.

\section{Calibration for Water Content}

The goal is to find the soil specific relation between measured dielectric constant (permittivity $\varepsilon^{\prime}$ ) and soil water content, the so-called calibration curve (see Fig. 4a). Whether this concerns volumetric or gravimetric water content is not that important. Next, one has to bear in mind that soil permittivity is dependent on soil density $(\rho)$, iron content, texture, organic matter content, temperature, and measurement frequency. Therefore, a calibration for soil water content is only valid for the specific soil used with it's specific properties. The accuracy with which soil water content can be measured depends on the calibration procedure and on the accuracy of the permittivity measurements. With some loss of accuracy, it is possible to use a calibration curve for soils with comparable properties. Now four ways to obtain water content values from permittivity measurements are discussed.

The easiest way is to buy equipment with pre-programmed calibration curves. This is only recommendable in case one can judge clearly from the specifications that the soil for which the calibration was made is comparable to ones own soil. Unfortunately this is hardly ever true. Comparing for example 'mineral soil' with 'mineral soil' is not advisable.

A second method is performing a two-point calibration. In general the permittivity at zero water content is about four or five. The shape of most calibration curves is similar to the famous Topp curve for sand (a third degree polynominal), but just rotated around the zero-point. One then has to make one additional permittivity measurement in a soil 
sample and determine it's water content The Topp equation can then be rotated until it fits the second point.

Third, to obtain a calibration curve based on more measurement points, one can use a cylinder to take an undisturbed soil sample. The cylinder should be large enough to not influence the measurement's electrical field lines and should be small enough to compact the soil too much. The soil in the column must be wetted carefully. A dielectric sensor is placed in the soil column, which then is placed on a balance. During the period that the soil column dries by air every now and then the sample weight and it's corresponding permittivity value are registered. Finally, the soil is oven dried and it's water content is determined. Together with the sample cylinders volume the calibration curve can be derived.

The last method is to make density dependent calibration curves to account for density variations in depth (theory by Perdok et al., 1996). In the laboratory soil sample cylinders are artificially compacted to a certain bulk density. This will be done for two series of different gravimetric water contents and for a series of different densities (see Fig. 4). Separately the equations for permittivity as a function of gravimetric water content, $\varepsilon^{\prime}(\mathrm{w})$, and for permittivity as a function of density, $\varepsilon^{\prime}(\rho)$, can be made. The shape of $\varepsilon^{\prime}(w)$ is a third degree polynominal, the shape of $\varepsilon^{\prime}(\rho)$ is a straight line. Further, the slope of the $\varepsilon^{\prime}(\rho)$ relation is linearly dependent on the water content (J. Kroesbergen, personal communication). Combining the above a soil specific $\varepsilon^{\prime}(w, \rho)$ equation can be derived. Now for each dielectric sensor to be installed in the soil specific calibration curve can be computed. When installing the sensor one determines the gravimetric water content at the point of installation and one performs a permittivity measurement, so with these values density can be calculated. The advantage of this method is that no time consuming core samples have to be taken.

Concerning the above four calibration methods one can say that the first method is the least accurate, because it is hardly ever soil specific enough, and the last method is the most accurate because it allows for density dependency. A two-point calibration is the fastest way. The balance method is less labour intensive than the density dependent method, but it covers a longer period because the soil has to dry by air. Before one decides which method to choose, one has to know what accuracy is desirable and what working range the calibration curve should cover. The curve's dry end $(<5-7 \%$ gravimetric water content) is a problem with dielectric measurements, due to not fully understood bound water effects. The same applies for the wet end.

\section{Temperature Dependence of Dielectric Constant and Bulk EC}

Both dielectric constant and bulk EC depend on temperature. We want to correct for this influence since the quantities that we are really interested in, water content and nutrient concentration, are not temperature dependent. First an impression is given on what order of magnitude the dependencies cover. Second the direction of research for finding correction formulas is discussed.

During a period in spring with no irrigation the maximum differences per day measured in our greenhouse sandy soil were: $\Delta \mathrm{T}_{\max }=7^{\circ} \mathrm{C}, \Delta \varepsilon^{\prime}{ }_{\max }=0.4, \Delta \mathrm{EC}_{\max }=0.03$ $\mathrm{mS} / \mathrm{cm}$ at $5 \mathrm{~cm}$ depth and $\Delta \mathrm{T}_{\max }=0.5^{\circ} \mathrm{C}, \Delta \varepsilon^{\prime}{ }_{\max }=0.1, \Delta \mathrm{EC}_{\max }=0.01 \mathrm{mS} / \mathrm{cm}$ at $45 \mathrm{~cm}$ depth (see Fig. 5). In the observed period no crops were grown and no nutrients were applied. Therefore the differences are not very large. The observed differences are not important when compared to sensor accuracy, but they are important when compared to sensor repeatability.

The temperature dependence was investigated as follows. First, through corresponding $\varepsilon$ ', EC and T lines a trendline was fitted. Then the difference of the $\varepsilon^{\prime}, \mathrm{EC}$ and $\mathrm{T}$ values compared to their trendline was calculated. Finally the calculated differences of $\varepsilon$ ' and $\mathrm{EC}$ were plotted individually against the calculated differences in T. The lines fitted through these plots crossed the origin for EC data only, indicating that when temperature does not change the $\mathrm{EC}$ value does not change. For $\varepsilon$ ' data no trendlines cross the origin, but cross at a lower point on the $\mathrm{x}$-axis. This means that soil water 
content decreased.

Future research concerning the temperature dependence will be based on the following assumptions. Since we know that the EC's temperature dependence of a material is dependent on the absolute $\mathrm{EC}$ value at a reference point according to:

$\mathrm{EC}=\mathrm{EC}_{\text {ref } \mathrm{T}}(1+0.0225 \cdot \Delta \mathrm{T})$

and we know that $\varepsilon^{\prime}$ and $\mathrm{EC}$ are linearly dependent on each other it seems likely that $\varepsilon^{\prime}$ is dependent on temperature the same way the EC is. However, there are two differences between the $\varepsilon^{\prime}(\mathrm{T})$ relation and $\mathrm{EC}(\mathrm{T})$ relations. First, the $\varepsilon^{\prime}(\mathrm{T})$ relation crosses the $\mathrm{xaxis}$ at zero water content $\varepsilon$ ' where the temperature influence is negligible, while the EC(T) relation crosses the origin. Second the $\varepsilon^{\prime}(T)$ relation will be for a constant water content, while the $\mathrm{EC}(\mathrm{T})$ will be for constant nutrient concentration.

\section{DISCUSSION AND CONCLUSIONS}

Once more it has been shown that developing an advanced control system demands a lot of knowledge about the process and how it can be monitored. The Waterman irrigation control system, presented as a concept in section 2.3, should be flexible enough to enable users to choose their own functionality of interest.

The article title 'One starts measuring soil moisture, one obtains data and then what?' (Vegter, 1995) clearly shows the problem when people want to start controlling irrigation based on soil moisture sensors. In general, no choice whether to measure pressure head, water content, or both is made purposefully. Solely measuring pressure head seems suitable for a wide range of applications, but for sandy soils and when using a ground water flow model one should consider measuring water content too.

The accuracy of calibration for soil water content will stay a point of concern as long as there are sensor manufacturers that claim unrealistic high accuracies. From the four methods presented, the Kroesbergen (Perdok et al., 1996) method seems favourable because it provides density dependent calibration formulas. The last remark on calibration is that for controlling purposes it is not important at what water content value the process is kept. The grower just needs a reproducible set point that is related to water content changes. Considering this, controlling the dielectric constant is sufficient. However, for comparing results of different nurseries with each other a water content value offers more clarity.

Temperature dependence correction formulas for $\varepsilon$ ' and EC should improve the accuracy of controlling soil water content and salinization level. Future research will be based on the similarities between $\varepsilon$ ' and EC.

\section{ACKNOWLEDGEMENTS}

The authors would like to thank the following financiers of the Waterman project for their contribution: the Ministry of Agriculture, Nature Management and Fisheries of the Netherlands (program 'Optimisation of water and nutrient conservation in protected cropping systems', no. 256) and the European Community (Fair-4 P0681).

\section{Literature Cited}

Anonymous, 1989. Stuctuurnota landbouw. Ministry of Agriculture, Nature Management and Fisheries. The Hague, the Netherlands. p77-79 and p135.

Anonymous, 1997. Denar's solide finish. Milieudemonstratie-project Denar Kas BV. CDROM produced by Vreugdenhil Producties, Ruys de Beerenbrouckplein 7, 2613 AR Delft, The Netherlands.

Boonekamp G., 1994. Consequent nat of droog is het beste. Groenten+Fruit, vakdeel glasgroenten 4(1994)19, p6-9.

Hess T. 1996. A microcomputer scheduling program for supplementary irrigation. Computers and Electronics in Agriculture 15:233-243.

Meron, M., Assaf, R., Bravdo, B., Wallach, R., Hallel, R., Levin, A and Dahan, I. 1995. 
Soil sensor actuated microirrigation of apples. In Proc. $5^{\text {th }}$ Int. Microirrigation Congress, 486-491. St. Joseph, Mich.: ASAE.

Perdok, U.D., Kroesbergen, B. and Hilhorst, M.A.. 1996. Influence of gravimetric water content and bulk density on the dielectric properties of soil. European journal of soil science, 47(3):367-371.

Reddy, J.M. 1985. Adaptive control in management of surface irrigation systems. ASAE Paper 85-2579.

Smith, R.J. and McClymont, D.J. 1996. Toward real time control of surface irrigation: estimation of soil infiltration parameters under surface and surge irrigation. Trans ASAE Paper 96C-053.

Thomson, S.J. and Ross, B.B. 1996. Model-based irrigation management using a dynamic parameter adjustment method. Computers and Electronics in Agriculture 14:269-290.

Valiente M., de Juan, J.A.,Tarjuelo, J. and Ortega, F. 1996. Model for optimum irrigation water management, application to an extensive horticulture farm. Proceedings of AgEng, paper 96C-040, p605-606.

Vegter, B. 1995. Je gaat vocht meten, je krijgt een cijfer, maar wat dan? Vakblad voor de Bloemisterij 12, p22-23.

\section{Figures}

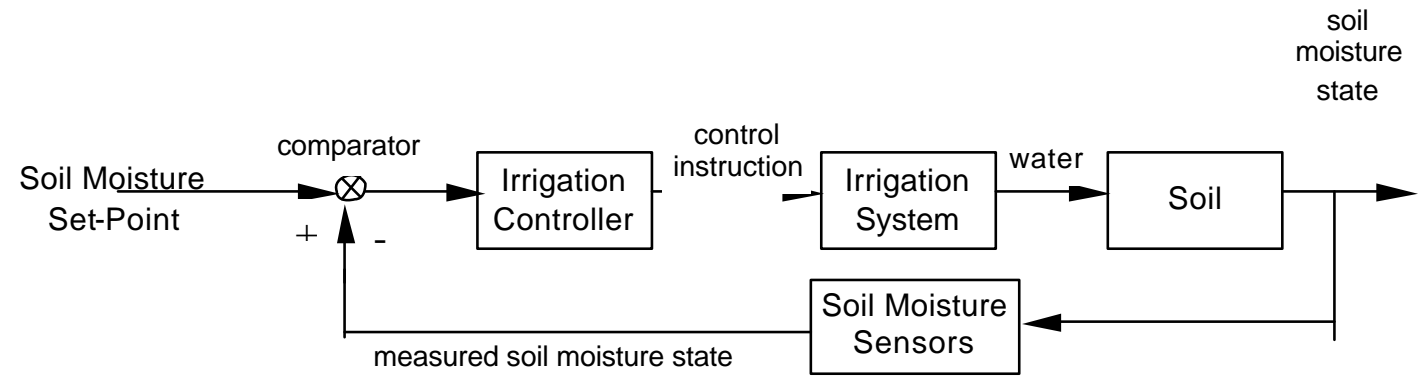

Fig. 1. Basic feedback control loop for irrigation control. 


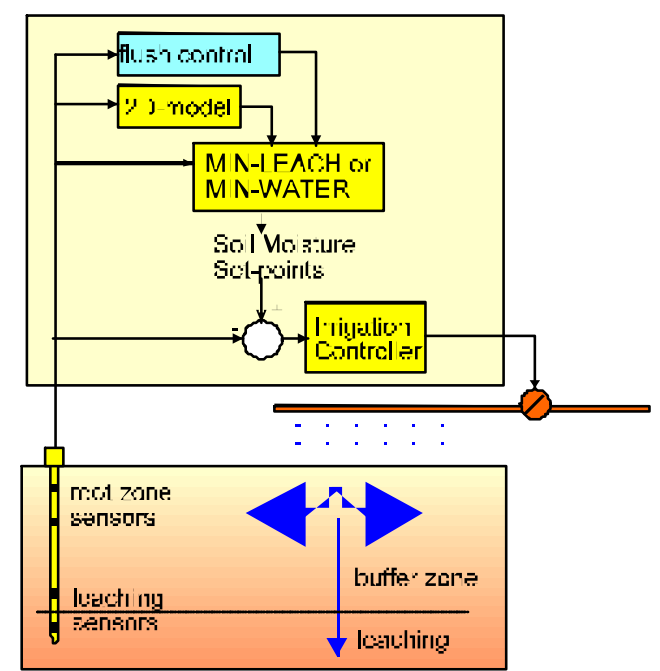

Fig. 2. Concept of the Waterman irrigation control system.

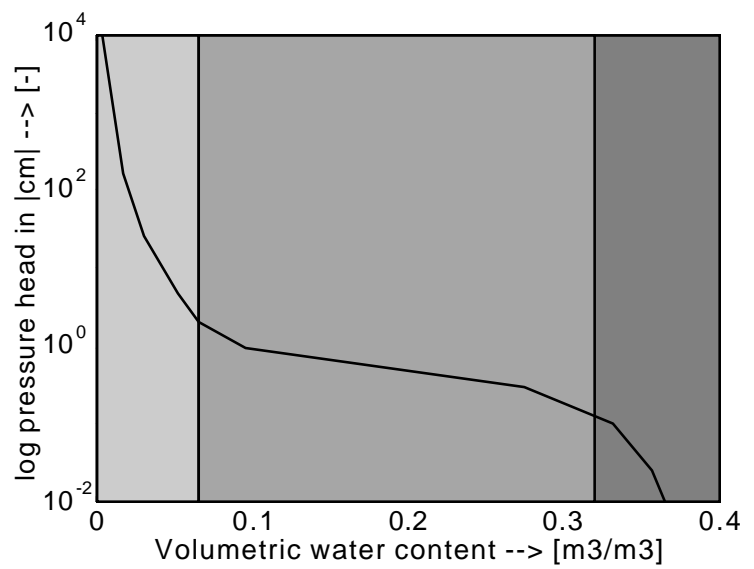

Fig. 3. Water retention curve of a medium course sandy soil. The light grey and the dark grey areas indicate soil moisture ranges with high resolution for pressure head measurements. Measurements of water content will have a low resolution. The medium grey area indicates the opposite. 

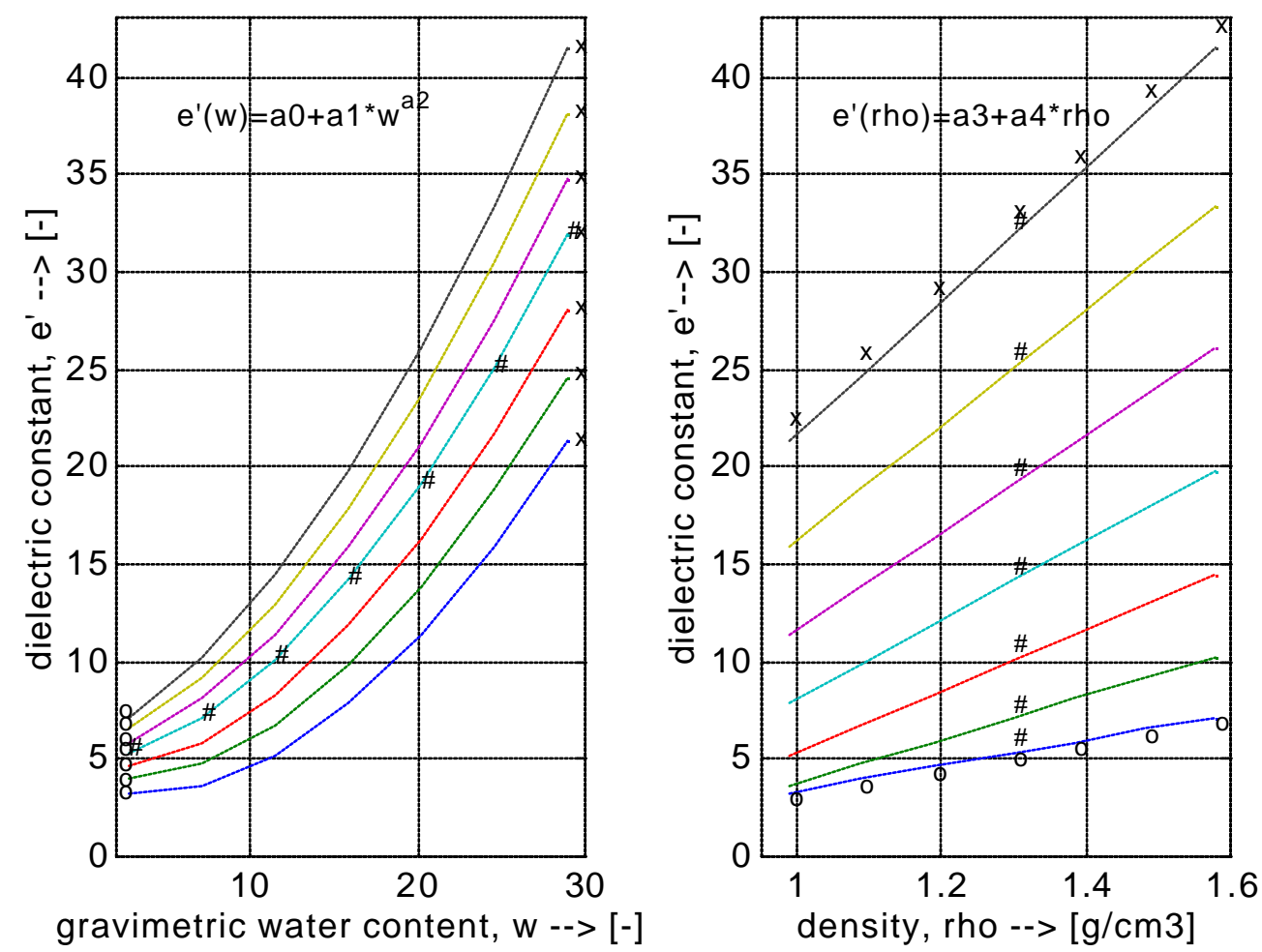

Fig. 4a and 4b. $\varepsilon^{\prime}(w)$ and $\varepsilon^{\prime}(\rho)$ calibration functions for a sandy soil. Theory from Perdok et al (1996). Each mark represents an individually compacted soil sample. The o's indicate a soil sample series of constant low water content and the x's indicate a series of constant high water content. The soil sample series with constant density is indicated by \#'s. 
a)

b)
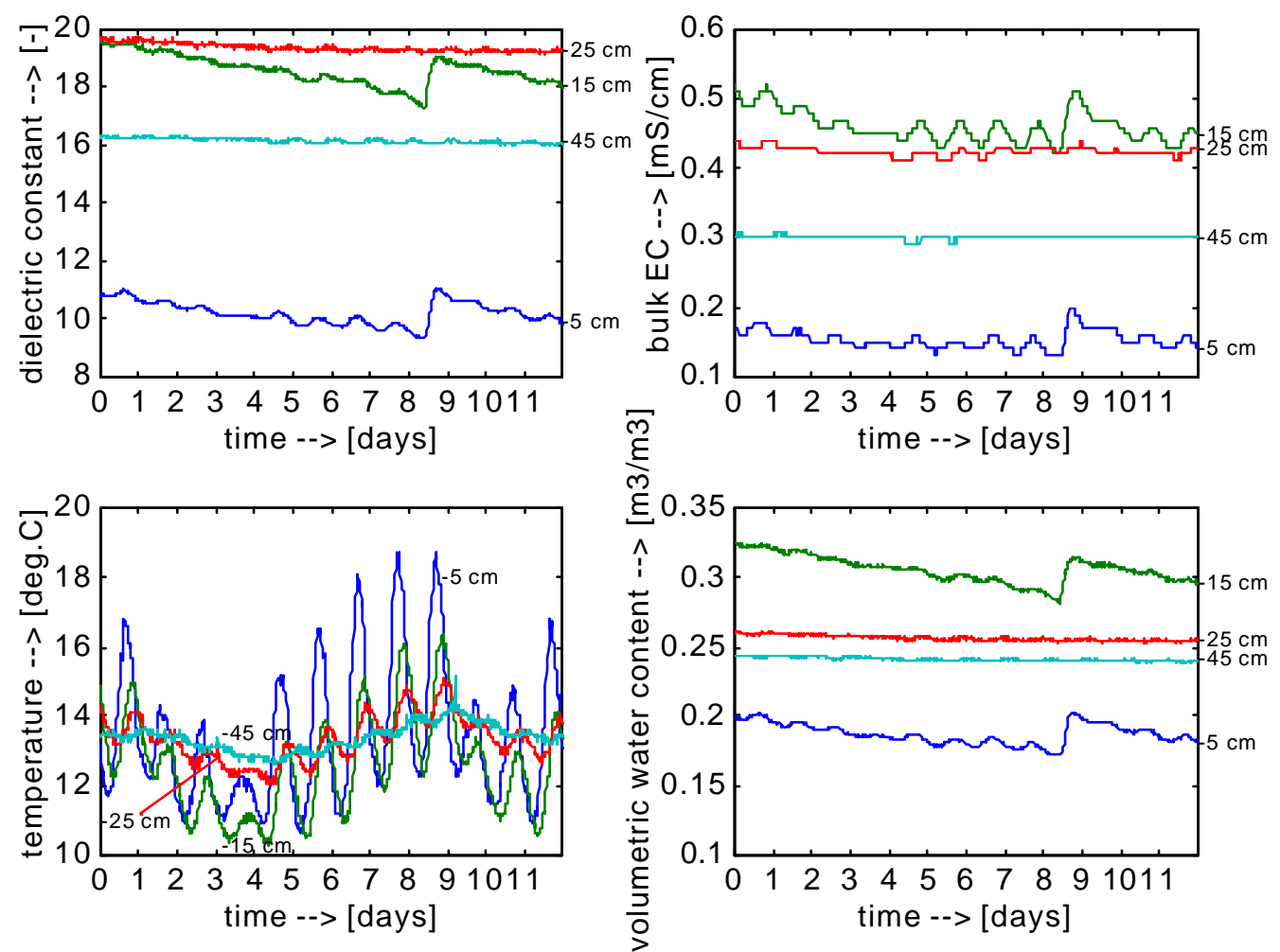

Fig. 5. Measurements of soil dielectric constant bulk EC and temperature, together with calculated volumetric water content. The measurements were performed in a sandy loam soil at four different depths from 1-4-'97 to 12-4-'97. Irrigation was applied on the eighth day. 